HUPD-9521

KEK Preprint 95-109

October, 1995

\title{
Lattice Heavy Quark Effective Theory and the Isgur-Wise function
}

\author{
Shoji Hashimoto \\ National Laboratory for High Energy Physics (KEK), \\ Tsukuba 305, Japan \\ and \\ Hideo Matsufuru \\ Department of Physics, Hiroshima University, \\ Higashi-Hiroshima 739, Japan
}

\begin{abstract}
We compute the Isgur-Wise function using heavy quark effective theory formulated on the lattice. The non-relativistic kinetic energy term of the heavy quark is included to the action as well as terms remaining in the infinite quark mass limit. The classical velocity of the heavy quark is renormalized on the lattice and we determine the renormalized velocity non-perturbatively using the energy-momentum dispersion relation. The slope parameter of the Isgur-Wise function at zero recoil is obtained at $\beta=6.0$ on a $24^{3} \times 48$ lattice for three values of $m_{Q}$.
\end{abstract}




\section{Introduction}

In the determination of the CKM (Cabibbo-Kobayashi-Maskawa) matrix element $\left|V_{c b}\right|$ from experiment of the exclusive decay $B \rightarrow D^{(*)} l \bar{\nu}$ the heavy quark symmetry plays an essential role, because the universal form factor $\xi\left(v \cdot v^{\prime}\right)$, so called the Isgur-Wise function, is normalized to be one at zero recoil in the infinite heavy quark mass limit. However the differential decay width for this process disappear at the zero recoil point, so that one needs to extrapolate the experimental data to this point. The lattice computation of the slope $\xi^{\prime}(1)$ enables this extrapolation in a model independent way. On the lattice Bernard, Shen and Soni [1] and the UKQCD Collaboration [2] have computed the Isgur-Wise function using the propagating quarks (Wilson fermion and clover fermion respectively) for the heavy quark. Since the heavy quark mass should be below the lattice cutoff in this framework, present simulations are performed around the charm quark mass. An alternative way of treating the heavy quark is to use the heavy quark effective theory (HQET) on the lattice. Mandula and Ogilvie formulated the Lattice HQET and applied it to a calculation of the Isgur-Wise function[3]. Their simulation, however, suffers from much noise so that the extraction of the ground state seems to be difficult. This problem is well known in the calculation of the heavy-light decay constant using the static approximation where one is forced to use some method to enhance the signal of the ground state [4 or to introduce $O\left(1 / m_{Q}\right)$ terms in order to decrease the statistical noise [5].

Our calculation is based on the lattice HQET keeping a part of the $O\left(1 / m_{Q}\right)$ correction terms. The inclusion of the kinetic term of the heavy quark reduces the statistical noise significantly and enables us to extract the ground state reliably. We calculate the Isgur-Wise function for three values of the heavy quark mass and discuss $O\left(1 / m_{Q}\right)$ effect on it.

The plan of the paper is as follows. Section 2 contains the formulation of the lattice HQET including $O\left(1 / m_{Q}\right)$ corrections. In Section 3 we describe

our numerical simulations and discuss the results. In this section, after brief account of simulation parameters, we first mention about the renormalization of classical velocity. Then the calculation of the Isgur-Wise function is described. Sec. 4 is devoted to the conclusion. 


\section{HQET on the lattice}

The action of the lattice HQET including $O\left(1 / m_{Q}\right)$ corrections is

$$
S_{h}=\sum_{x} \bar{h}(x)\left[-i v \cdot D-\frac{1}{2 m_{Q}}\left(D^{2}+\frac{1}{2} \sigma_{\mu \nu} F_{\mu \nu}\right)\right] h(x)
$$

where $v_{\mu}=\left(\vec{v}, i v^{0}\right)$ is a four-velocity of the moving heavy quark. $D_{\mu}$ is a lattice covariant derivative and $F_{\mu \nu}$ is a chromo-magnetic (electric) field. This action is a generalization of the non-relativistic lattice QCD (NRQCD) for finite velocities. The first term corresponds to the action in the infinite mass limit used by Mandula and Ogilvie [3] and the second term describes the $O\left(1 / m_{Q}\right)$ corrections. The heavy quark field $Q(x)$ is expressed in terms of $h(x)$ as

$$
Q(x)=e^{i m_{Q} v \cdot x}\left[1-\frac{1}{2 m_{Q}} \not D_{\perp}\right] h(x),
$$

where $D_{\perp \mu}=D_{\mu}+v_{\mu}(v \cdot D)$. For simplicity we neglect the spin dependent interaction term $\frac{1}{4 m_{Q}} \sigma_{\mu \nu} F_{\mu \nu}$ from the action and the $O\left(1 / m_{Q}\right)$ correction term from the field redefinition, i.e. the second term in eq. (2). It should be noted that the $O\left(1 / m_{Q}\right)$ corrections are not included completely with this approximation, but the kinetic term describes the motion of the heavy quark inside the meson and gives a major $O\left(1 / m_{Q}\right)$ effect.

The heavy quark propagator is obtained by solving an evolution equation

$$
\begin{aligned}
G(x+\hat{t})= & U_{4}^{\dagger}(x)\left(1-\frac{1}{n} H\right)^{n} G(x) \\
& +(\text { source term })
\end{aligned}
$$

and

$$
H=\frac{1}{v^{0}}\left\{-i \vec{v} \cdot \vec{D}-\frac{1}{2 m_{Q}}\left(\frac{-1}{\left(v^{0}\right)^{2}}(\vec{v} \cdot \vec{D})^{2}+\vec{D}^{2}\right)\right\}
$$

where we used the equation of motion in the infinite mass limit

$$
\left(v^{0} D_{4}-i \vec{v} \cdot \vec{D}\right) h(x)=0
$$

to remove the $D_{4}^{2}$ term from the evolution equation. $D_{i} h(x)$ and $D_{i}^{2} h(x)$ are defined as

$$
\begin{aligned}
D_{i} h(x) & =\frac{U_{i}(x) h(x+\hat{i})-U_{i}^{\dagger}(x-\hat{i}) h(x-\hat{i})}{2} \\
D_{i}^{2} h(x) & =U_{i}(x) h(x+\hat{i})+U_{i}^{\dagger}(x-\hat{i}) h(x-\hat{i})-2 h(x) .
\end{aligned}
$$


The cost for the computation of this deterministic equation is much less than one of obtaining the propagator for the Wilson fermion using some iterative solver. A parameter $n$ is introduced to stabilize unphysical high frequency modes. Considering the evolution equation for free field, the stability condition to assure the convergence of the equation is

$$
\begin{gathered}
\left|1-\frac{1}{n} H_{0}(\vec{k})\right|<1 \quad(\text { for any } \vec{k}), \\
H_{0}(k)=\frac{1}{v^{0}}\left\{\vec{v} \cdot \overrightarrow{\hat{k}}+\frac{1}{2 m_{Q}}\left(\overrightarrow{\hat{k}}^{2}-\left(\frac{\vec{v}}{v^{0}} \cdot \overrightarrow{\hat{k}}\right)^{2}\right)\right\},
\end{gathered}
$$

where $\tilde{k}_{i}=\sin k_{i}$ and $\hat{k}_{i}=2 \sin \frac{k_{i}}{2}$. According to the definition of derivatives (6), $\hat{k}$ in the last term of (8) should be $\tilde{k}$. We, however, use $\hat{k}$ instead of $\tilde{k}$ because the effect of this term is small for small $\vec{v}$, and for convenience in later calculation. Similar condition is required in NRQCD [6], but in the present case it depends on the heavy quark velocity as well as $m_{Q}$ and $n$. Because of the presence of term $\frac{1}{v^{0}} \vec{v} \cdot \overrightarrow{\vec{k}}$ high frequency modes of residual momentum do not converge with time evolution for a large value of velocity unless $n$ is taken to be sufficiently large. On the other hand the $O(a)$ effect becomes larger for larger $n$, then much large $n$ is not preferable. Fixing $n$ for a value of $m_{Q}$, magnitude of velocities are restricted below a certain value. For the values of parameter used in the present simulation, $\sum_{i}\left|v_{i}\right|$ is restricted to be smaller than 0.25 .

Multiplying the projection operator to $G(x)$, the heavy quark propagator in 4-spinor representation is obtained as

$$
S_{Q}(x)=G(x) \otimes \frac{1-i \not b}{2} .
$$

The Isgur-Wise function is extracted from the three point correlation function

$$
\left\langle 0\left|B\left(v^{\prime}\right) V_{4}\left(v^{\prime}, v\right) B(v)^{\dagger}\right| 0\right\rangle
$$

where $V_{4}\left(v^{\prime}, v\right)$ is a temporal component of the vector current $\bar{h}_{v^{\prime}} \gamma_{4} h_{v}$ and $B(v)$ is a local interpolating field for an initial or a final meson state $\bar{q} \gamma_{5} h_{v}$ for which we take the same mass for both initial and final mesons. The three point correlation function is related to the Isgur-Wise function $\xi\left(v \cdot v^{\prime}\right)$ as

$$
G_{v \rightarrow v^{\prime}}\left(t_{f}, t_{s}, t_{i}\right)=\sum_{\vec{x}_{s}} \sum_{\vec{x}_{f}}\left\langle 0\left|B\left(v^{\prime} ; x_{f}\right) V_{4}\left(v^{\prime}, v ; x_{s}\right) B^{\dagger}\left(v ; x_{i}\right)\right| 0\right\rangle
$$




$$
\begin{aligned}
& =\sum_{\vec{x}_{s}} \sum_{\vec{x}_{f}}\left\langle\operatorname{Tr}\left[S_{q}\left(x_{f}, x_{i}\right)^{\dagger} S_{h_{v^{\prime}}}\left(x_{f}, x_{s}\right) \gamma_{4} S_{h_{v}}\left(x_{s}, x_{i}\right)\right]\right\rangle \\
& \propto m_{B} \xi\left(v \cdot v^{\prime}\right)\left(v+v^{\prime}\right)_{0} \\
& \times e^{-E_{f}\left(t_{f}-t_{s}\right)-E_{i}\left(t_{s}-t_{i}\right)} \quad\left(\text { for } t_{f} \gg t_{s} \gg t_{i}\right)
\end{aligned}
$$

where $x_{f}=\left(\vec{x}_{f}, t_{f}\right), x_{s}=\left(\vec{x}_{s}, t_{s}\right), x_{i}=\left(0, t_{i}\right)$ and the component proportional to $\left(v-v^{\prime}\right)_{0}$ is neglected since its form factor is $O\left(\Lambda_{Q C D} / m_{Q}\right)$ and $\left(v-v^{\prime}\right)_{0}$ is small itself in our velocity region. Taking the following ratio of the threepoint functions the exponential factor and the renormalization factor for the vector current cancel and we obtain

$$
\begin{aligned}
R_{v, v^{\prime}}\left(t_{f}, t_{s}, t_{i}\right) & =\frac{G_{v \rightarrow v^{\prime}}\left(t_{f}, t_{s}, t_{i}\right) G_{v^{\prime} \rightarrow v}\left(t_{f}, t_{s}, t_{i}\right)}{G_{v \rightarrow v}\left(t_{f}, t_{s}, t_{i}\right) G_{v^{\prime} \rightarrow v^{\prime}}\left(t_{f}, t_{s}, t_{i}\right)} \\
& \rightarrow\left|\xi\left(v \cdot v^{\prime}\right)\right|^{2} \frac{\left(v_{0}+v_{0}^{\prime}\right)^{2}}{4 v_{0} v_{0}^{\prime}} .
\end{aligned}
$$

We use this relation for the calculation of the Isgur-Wise function.

\section{Simulations and results}

We used 120 gauge configurations of $24^{3} \times 48$ lattice at $\beta=6.0$ in the quenched approximation. Each configurations are separated by 2,000 pseudoheat bath sweeps after 20,000 sweeps for thermalization. For the light quarks we used the Wilson fermion with hopping parameters 0.153 and 0.155 , and obtained propagators under the periodic and Diriclet boundary conditions for spatial and temporal direction respectively. The critical hopping parameter and the inverse lattice spacing determined from rho meson mass are $\kappa_{c}=$ $0.156986(50)$ and $a^{-1}=2.215(64) \mathrm{GeV}$.

Heavy quark masses and stabilization parameters we used are

$$
\left(\begin{array}{c}
m_{Q} \\
n
\end{array}\right)=\left(\begin{array}{c}
1.8 \\
3
\end{array}\right),\left(\begin{array}{c}
2.5 \\
2
\end{array}\right),\left(\begin{array}{c}
5.0 \\
1
\end{array}\right)
$$

where $m_{Q}=1.8$ nearly corresponds to the bottom quark mass. As mentioned above, heavy quark velocities should satisfy the stability condition (8) which leads $\sum_{i}\left|v_{i}\right| \leq 0.25$ for our sets of $m_{Q}$ and $n$. In Table 1 we tabulate our sets of $v$ and $v^{\prime}$ used in the simulation. 
The mean-field improvement [7] is applied when we compute the heavy quark evolution equation. Link variables are altered as $U_{\mu}(x) \rightarrow U_{\mu}(x) / u_{0}$ where $u_{0}=\left\langle\frac{1}{3} U_{\text {plaq. }}\right\rangle^{1 / 4}$. $U_{\text {plaq. }}$ is the product of link variables along plaquette. We used the value $u_{0}=0.8776$ measured on our configurations.

\subsection{Extraction of the renormalized velocity}

Before we proceed to the calculation of the Isgur-Wise function we mention about the determination of masses and velocities of the heavy-light mesons. The velocity of the moving heavy-light meson $v_{R}$ could differ from the one of the bare heavy quark $v$ for the lattice HQET because of the violation of the space-time $O(4)$ symmetry $[8,9]$. We extract the 'renormalized' velocity from the simulation results nonperturbatively using the dispersion relation of the meson.

The heavy-light meson obeys the dispersion relation

$$
p^{0}=m_{P} v^{0}+\frac{1}{v^{0}}\left\{\vec{v} \cdot \vec{k}^{\prime}+\frac{1}{2 m_{P}}\left({\overrightarrow{k^{\prime}}}^{2}-\left(\frac{\vec{v}}{v^{0}} \cdot{\overrightarrow{k^{\prime}}}^{2}\right)\right\}\right.
$$

where $p^{\mu}$ is the meson momentum which is decomposed as $p^{\mu}=m_{P} v^{\mu}+k^{\prime \mu}$. $k_{\mu}^{\prime}$ differs from our definition of the residual momentum $k_{\mu}$ which is defined through $p^{\mu}-m_{Q} v^{\mu}$. Using a naive mass relation

$$
m_{P}=m_{Q}+E_{0}
$$

where $E_{0}$ is the binding energy, $k^{\prime \mu}$ is written as $k^{\prime \mu}=k^{\mu}-E_{0} v^{\mu}$. Substituting this relation in above dispersion relation, we obtain

$k^{0}=\frac{E_{0}}{v^{0}}\left(1+\frac{E_{0}}{2 m_{P}}\left(\frac{\vec{v}}{v^{0}}\right)^{2}\right)+\frac{\vec{v}}{v^{0}} \cdot \vec{k}\left(1-\frac{E_{0}}{m_{P} v^{0^{2}}}\right)+\frac{1}{2 m_{P} v^{0}}\left(\vec{k}^{2}-\left(\frac{\vec{v}}{v^{0}} \cdot \vec{k}\right)^{2}\right)$.

From now on, we write $E(k)$ instead of $k^{0}$, and attach subscript ' $\mathrm{R}$ ' to the classical velocity $v$ for distinction from ones as the input parameter.

On the lattice, above dispersion relation is rewritten as

$$
e^{-[E(k)-E(k=0)]}=1-\left\{\frac{\overrightarrow{v_{R}}}{v_{R}^{0}} \cdot\left(\overrightarrow{\tilde{k}}-\frac{E_{0}}{m_{P} v_{R}^{0}} \overrightarrow{\hat{k}}\right)+\frac{1}{2 m_{P} v_{R}^{0}}\left(\overrightarrow{\hat{k}}^{2}-\left(\frac{\overrightarrow{v_{R}}}{v_{R}^{0}} \cdot \overrightarrow{\hat{k}}\right)^{2}\right)\right\},
$$


where

$$
E(k=0)=\frac{E_{0}}{v_{R}^{0}}\left(1+\frac{E_{0}}{2 m_{P}}\left(\frac{\overrightarrow{v_{R}}}{v_{R}^{0}}\right)^{2}\right) .
$$

In Fig. 1 1 we plot the asymmetry of the energy $-\left[e^{-E(k)+E(0)}-e^{-E(-k)+E(0)}\right] / 2$ from which we extract the 'renormalized' velocity $v_{R}$ using the relation

$$
-\frac{e^{-E(k)+E(0)}-e^{-E(-k)+E(0)}}{2}=\frac{v_{R i}}{v_{R}^{0}} \cdot\left(\sin k_{i}-\frac{E_{0}}{m_{P} v_{R}^{0}} 2 \sin \frac{k_{i}}{2}\right) .
$$

For $E_{0}$ and $m_{P}$ in the right hand side of (18), we use the values obtained at $\vec{v}=0$. For the value of $v^{0}$, bare one is used. These substitution should be justified by the independence of these values on the velocity. Obtained values of the 'renormalized' velocity are listed in Table 2 and slightly larger than 'bare' values $v$, but in most cases consistent with $v$ in present error level. Ratios $v_{R} / v$ are almost independent on $v$ as shown in Fig. 2.

The heavy-light meson masses can also be obtained similarly using the relation

$$
1-\frac{e^{-E(k)+E(0)}+e^{-E(-k)+E(0)}}{2}=\frac{1}{2 m_{P} v_{R}^{0}}\left(\overrightarrow{\hat{k}}^{2}-\left(\frac{\vec{v}_{R}}{v_{R}^{0}} \cdot \overrightarrow{\hat{k}}\right)^{2}\right)
$$

which we plot in Fig. 3. The obtained values for $m_{P}$ are summarized in Table 3. In Table 4 , we also list the results for binding energy of heavy-light meson, $E_{0}$, determined from correlation function at zero residual momentum using equation (17). In eq. (17), we again use the values at $\vec{v}=0$ for $E_{0}$ and $m_{P}$. Both of $m_{P}$ and $E_{0}$ are almost independent of the velocity and well satisfy the naive mass relation $m_{P}=m_{Q}+E_{0}$.

\subsection{The Isgur-Wise function}

In the calculation of the three point function we set $t_{i}=8$ and $t_{s}=20$ where it seems to reach the ground state of initial meson moving with velocity $v$. Fig. 4 shows the behavior of $R_{v, v^{\prime}}\left(t_{f}, t_{s}, t_{i}\right)$ as functions of $t_{f}$ for several sets of $v$ and $v^{\prime}$ for $m_{Q}=1.8$ and $\kappa=0.153$. Signal is rather clean and we observe clear plateaus beyond $t_{f} \approx 24$. Then we take $t_{f}=24-27$ as a fitting interval. For the largest mass $m_{Q}=5.0$ signal becomes so noisy and we cannot identify a clear plateau. Nevertheless we treat them in the same manner as $m_{Q}=1.8$ and 2.5 with $t_{f}=24-25$. 
Our results for the form factor $\xi\left(v_{R} \cdot v_{R}^{\prime}\right)$ at $\kappa=0.153$ are shown in Fig. 5 for $m_{Q}=1.8,2.5$ and 5.0. Error bars in the horizontal direction are coming from the statistical uncertainty in the determination of the renormalized velocity. Statistical errors increase with heavy quark mass as expected. In our $v \cdot v^{\prime}$ region the form factors have almost linear behavior where we extract the slope of $\xi\left(v_{R} \cdot v_{R}^{\prime}\right)$ at $v_{R} \cdot v_{R}^{\prime}=1$, which is usually denoted as $-\rho^{2}$, from a fit of our data to the the form

$$
\xi\left(v_{R} \cdot v_{R}^{\prime}\right)=1-\rho^{2}\left(v_{R} \cdot v_{R}^{\prime}-1\right) .
$$

$\rho^{2}$ for each $m_{Q}$ and $\kappa$ are given in Table 5 with the values extrapolated to the critical hopping parameter for the light quark. $m_{Q}=1.8$ roughly corresponds to the $\mathrm{B}$ meson mass where our data should be compared with the experimental data obtained by CLEO Collaboration 10.

$$
\begin{array}{rll}
\rho^{2} & =0.70 \pm 0.17 \quad \text { this work } \\
& =0.84 \pm 0.15 \quad \text { CLEO }
\end{array}
$$

Our data is consistent with that of CLEO considering the large statistical uncertainty. We also compare our results with other lattice results obtained using the propagating heavy quarks for which the heavy quark mass is around the charm quark mass

$$
\begin{aligned}
\rho^{2} & =1.24 \pm 0.26 \text { (stat) } \pm 0.33 \text { (syst) } \\
& =0.9_{-3}^{+2}(\text { stat })_{-2}^{+4} \text { (syst) }
\end{aligned}
$$

These are again consistent with each other in their large statistical uncertainty.

In order to see the $O\left(1 / m_{Q}\right)$ effect we extrapolate our $\rho^{2}$ to the vanishing $1 / m_{Q}$ limit as shown in Fig. 6, $\rho^{2}$ becomes smaller when we approach the infinite mass limit. We need, of course, more statistics to quantify the size of the $O\left(1 / m_{Q}\right)$ effect and to extrapolate our data to the charm quark mass.

\section{Conclusion}

We calculated the Isgur-Wise function near the zero recoil limit using HQET including the kinetic term of the heavy quark. Spin-independent $O\left(1 / m_{Q}\right)$ 
effect is included in this approximation. The obtained value for the slope parameter $\rho^{2}$ is consistent with the experimental value, but the statistical error is still large. A large amount of this statistical error is due to the large statistical uncertainty in the determination of the renormalized classical velocity and it originates in extraction of binding energy of heavy-light meson for finite residual momenta. It is essential for reducing the statistical error to improve the signal for finite momenta using the smearing techinique for example.

It is important to include the $O\left(1 / m_{Q}\right)$ terms completely and to quatify the size of violations of the heavy quark symmetry in several semi-leptonic form factors. The lattice HQET is also applicable for studies of heavy to light semi-leptonic decay ( $B \rightarrow \rho l \nu$ etc.) which can be used for extraction of $V_{u b}$ from the exclusive decay width in a model independent way.

\section{Acknowledgment}

The numerical computations were carried out on Intel Paragon XP/S at INSAM (Institute for Numerical Simulations and Applied Mathematics) of Hiroshima University. We would like to thank S. Hioki and O. Miyamura for

useful discussions. S.H. was supported in part by the Grant-in-Aid of the Ministry of Education under the contract No. 076117.

\section{References}

[1] C. Bernard, Y. Shen, and A. Soni, Phys. Lett. B317 (1993) 164.

[2] UKQCD Collaboration (K.C. Bowler et al.), hep-ph/9504231.

[3] J. Mandula and M. C. Ogilvie, Phys. Rev. D45 (1992) 2183 and heplat/9408006.

[4] C. Bernard, Nucl. Phys. B (Proc. Suppl.) 34 (1994) 47.

[5] S. Hashimoto, Phys. Rev. D50 (1994) 4639.

[6] B. A. Thacker and G. P. Lepage, Phys. Rev. D43 (1991) 196.

[7] G. P. Lepage and P. B. Mackenzie, Phys. Rev. D48 (1993) 2250. 
[8] U. Aglietti, Nucl. Phys. B421 (1994) 191.

[9] J. Mandula and M. C. Ogilvie, Nucl. Phys.B (Proc. Suppl.) 42 (1995) 424.

[10] CLEO Collaboration, Phys. Rev. D51 (1995) 1014. 


\section{TABLES}

\begin{tabular}{|c|c|}
\hline$\vec{v}$ ( initial) & \multicolumn{1}{|c|}{$\vec{v}^{\prime} \quad$ final $)$} \\
\hline \hline$(0,0,0)$ & $\begin{array}{c}\left(0,0, v_{f}\right) \\
v_{f}=0.05,0.10,0.15,0.20,0.25 \\
\left(0, v_{f}, v_{f}\right)\end{array}$ \\
& $v_{f}=0.05,0.10$ \\
\hline $\begin{array}{c}\left(0,0, v_{i}\right) \\
v_{i}=0.05,0.10,0.15,0.20,0.25 \\
\left(0,0, \pm v_{f}\right),\left(0, v_{f}, 0\right) \\
v_{f}=0.0,0.05,0.10,0.15,0.20,0.25 \\
\left(0, v_{f}, v_{f}\right),\left(0, v_{f},-v_{f}\right) \\
v_{f}=0.05,0.10\end{array}$ \\
\hline$\left(0, v_{i}, v_{i}\right) \quad v_{i}=0.05,0.10$ \\
$\begin{array}{c}\left(0, v_{f}, 0\right)\left(0,0,-v_{f}\right) \\
v_{f}=0.0,0.05,0.10,0.15,0.20,0.25 \\
\left(0, v_{f}, \pm v_{f}\right),\left(0,-v_{f},-v_{f}\right) \\
v_{f}=0.05,0.10\end{array}$ \\
\hline
\end{tabular}

Table 1: Initial and final heavy quark velocities used in our simulation. When we take the ratio $R_{v, v^{\prime}}\left(t_{f}, t_{s}, t_{i}\right)$ rotationally equivalent combinations of $v$ and $v^{\prime}$ are used. 


\begin{tabular}{|c||c|c|c|c|c|}
\hline \multicolumn{1}{|c||}{$\kappa=0.153$} & \multicolumn{5}{c|}{ bare velocity } \\
\hline$m_{Q}$ & 0.05 & 0.10 & 0.15 & 0.20 & 0.25 \\
\hline 1.8 & $0.054(04)$ & $0.105(05)$ & $0.157(07)$ & $0.209(10)$ & $0.263(14)$ \\
2.5 & $0.056(04)$ & $0.108(06)$ & $0.161(08)$ & $0.216(12)$ & $0.271(17)$ \\
5.0 & $0.059(05)$ & $0.112(08)$ & $0.166(12)$ & $0.222(20)$ & $0.279(31)$ \\
\hline \hline \multicolumn{1}{|c||}{$\kappa=0.155$} & \multicolumn{5}{|c|}{ bare velocity } \\
\hline$m_{Q}$ & 0.05 & 0.10 & 0.15 & 0.20 & 0.25 \\
\hline 1.8 & $0.052(06)$ & $0.102(07)$ & $0.152(09)$ & $0.204(12)$ & $0.255(16)$ \\
2.5 & $0.054(06)$ & $0.104(07)$ & $0.156(10)$ & $0.208(14)$ & $0.262(21)$ \\
5.0 & $0.055(07)$ & $0.107(10)$ & $0.158(15)$ & $0.210(23)$ & $0.261(35)$ \\
\hline
\end{tabular}

Table 2: Results for renormalized velocities for each $\kappa$ and $m_{Q}$. These values are obtained from simulation data nonperturbatively using the dispersion relation.

\begin{tabular}{|c||c|c|c|c|c|c|}
\hline \multicolumn{1}{|c||}{$\kappa=0.153$} & \multicolumn{7}{c|}{ bare velocity } \\
\hline$m_{Q}$ & 0.00 & 0.05 & 0.10 & 0.15 & 0.20 & 0.25 \\
\hline 1.8 & $2.30(06)$ & $2.28(06)$ & $2.26(07)$ & $2.24(09)$ & $2.21(12)$ & $2.17(16)$ \\
2.5 & $2.98(08)$ & $2.94(09)$ & $2.90(11)$ & $2.85(15)$ & $2.78(20)$ & $2.68(28)$ \\
5.0 & $5.39(21)$ & $5.31(24)$ & $5.17(33)$ & $4.99(51)$ & $4.81(82)$ & $4.72(134)$ \\
\hline \hline \multicolumn{1}{|c||}{$\kappa=0.155$} & \multicolumn{7}{|c|}{ bare velocity } \\
\hline$m_{Q}$ & 0.00 & 0.05 & 0.10 & 0.15 & 0.20 & 0.25 \\
\hline 1.8 & $2.30(07)$ & $2.29(08)$ & $2.27(09)$ & $2.26(12)$ & $2.24(15)$ & $2.20(21)$ \\
2.5 & $3.00(10)$ & $2.97(11)$ & $2.93(14)$ & $2.89(19)$ & $2.83(25)$ & $2.74(35)$ \\
5.0 & $5.45(25)$ & $5.40(29)$ & $5.28(40)$ & $5.16(61)$ & $5.09(100)$ & $5.20(169)$ \\
\hline
\end{tabular}

Table 3: The kinetic mass of heavy-light meson for each $m_{Q}$ and $\kappa$ obtained using the dispersion relation. Obtained values are almost independent of velocity. 


\begin{tabular}{|c||c|c|c|c|c|c|}
\hline \multicolumn{1}{|c||}{$\kappa=0.153$} & \multicolumn{7}{c|}{ bare velocity } \\
\hline$m_{Q}$ & 0.00 & 0.05 & 0.10 & 0.15 & 0.20 & 0.25 \\
\hline 1.8 & $0.531(04)$ & $0.530(04)$ & $0.529(04)$ & $0.528(04)$ & $0.527(05)$ & $0.525(06)$ \\
2.5 & $0.533(04)$ & $0.532(04)$ & $0.532(05)$ & $0.531(05)$ & $0.530(06)$ & $0.528(08)$ \\
5.0 & $0.533(06)$ & $0.534(06)$ & $0.535(08)$ & $0.537(10)$ & $0.539(13)$ & $0.544(19)$ \\
\hline \hline$\kappa=0.155$ & \multicolumn{7}{|c|}{ bare velocity } \\
\hline$m_{Q}$ & 0.00 & 0.05 & 0.10 & 0.15 & 0.20 & 0.25 \\
\hline 1.8 & $0.497(05)$ & $0.497(05)$ & $0.496(05)$ & $0.495(06)$ & $0.493(06)$ & $0.491(07)$ \\
2.5 & $0.500(06)$ & $0.499(06)$ & $0.499(06)$ & $0.498(07)$ & $0.496(08)$ & $0.495(10)$ \\
5.0 & $0.500(09)$ & $0.502(09)$ & $0.503(10)$ & $0.505(12)$ & $0.509(17)$ & $0.514(25)$ \\
\hline
\end{tabular}

Table 4: The binding energy obtained from correlation functions at zero residual momentum for each velocity, $m_{Q}$ and $\kappa$. Obtained values are almost independent of velocity.

\begin{tabular}{|c||c|c|c|c|}
\hline \multicolumn{1}{|c||}{$\kappa$} & \multicolumn{4}{c|}{$m_{Q}$} \\
\cline { 2 - 5 }$\kappa$ & 1.8 & 2.5 & 5.0 & $\infty$ \\
\hline 0.153 & $0.71(09)$ & $0.72(12)$ & $0.77(30)$ & $0.78(30)$ \\
0.155 & $0.70(13)$ & $0.71(18)$ & $0.77(47)$ & $0.75(46)$ \\
$\kappa_{c}$ & $0.70(17)$ & $0.69(24)$ & $0.77(66)$ & $0.73(67)$ \\
\hline
\end{tabular}

Table 5: Results for $\rho^{2} \equiv-\xi^{\prime}(1)$ for each $m_{Q}$ and $\kappa$. They were obtained by fitting $1-\rho^{2}\left(v_{R} \cdot v_{R}^{\prime}-1\right)$ with simulation data. Results at $m_{Q}=\infty$ are obtained from extrapolation in $1 / m_{Q}$ and the values at the critical hopping parameter $\left(\kappa_{c}=0.156986(50)\right)$ are obtained by linear extrapolation in $1 / \kappa$. 
Figure 1: Extraction of renormalized velocity at $m_{Q}=1.8$ and $\kappa=0.153$. It shows $-\left[e^{-E(k)+E(0)}-e^{-E(-k)+E(0)}\right] / 2$ vs $\sin \left(k_{z}\right)-\left(E_{0} / m_{P} v^{0^{2}}\right) 2 \sin \left(k_{z} / 2\right)$ for $v=(0,0,0.10)$ and $(0,0,0.20)$, where we use the obtained values at $\vec{v}=0$ for $E_{0}$ and $m_{P}$ and bare values for $v^{0}$. Solid lines represent linear fits from which we extract the renormalized velocities.

Figure 2: $\delta v / v$ for $m_{Q}=1.8$ and $\kappa=0.153 . \quad \delta v=v_{R}-v$ where the values of $v_{R}$ are listed in Table 2. $\delta v / v$ are independent of $v$ and slightly larger than zero, but consistent with zero in present error level.

Figure 3: $1-\left[e^{-E(k)+E(0)}+e^{-E(-k)+E(0)}\right] / 2$ as a function of $\left(2 \sin \left(k_{z}\right)\right)^{2}$ for $v=(0,0,0.20)$ at $m_{Q}=1.8$ and $\kappa=0.153$. Solid line represents linear fit from which we extract the kinetic mass of heavy-light meson.

Figure 4: $\quad R_{v, v^{\prime}}\left(t_{f}, t_{s}, t_{i}\right)$ as functions of $t_{f}$ for several sets of $v$ and $v^{\prime}$ at $m_{Q}=1.8$ and $\kappa=0.153$. For all combinations, $v^{\prime}$ s have the same absolute values as $v$ and opposite spatial directions to $v$.

Figure 5: The form factors $\xi\left(v_{R} \cdot v_{R}^{\prime}\right)$ at $m_{Q}=1.8(\mathrm{a}), 2.5$ (b) and 5.0 (c) where $\kappa=0.153$ for all cases. Figures show the almost linear behavior of $\xi\left(v_{R} \cdot v_{R}^{\prime}\right)$ in our velocity region. Slope of these functions, $\rho^{2}$, are extracted by fitting to the form $1-\rho^{2}\left(v_{R} \cdot v_{R}^{\prime}-1\right)$.

Figure 6: Extrapolation of $\rho^{2}$ to the vanishing $1 / m_{Q}$ limit at $\kappa=0.153$. The values of $\rho^{2}$ are listed in Table 5 . 


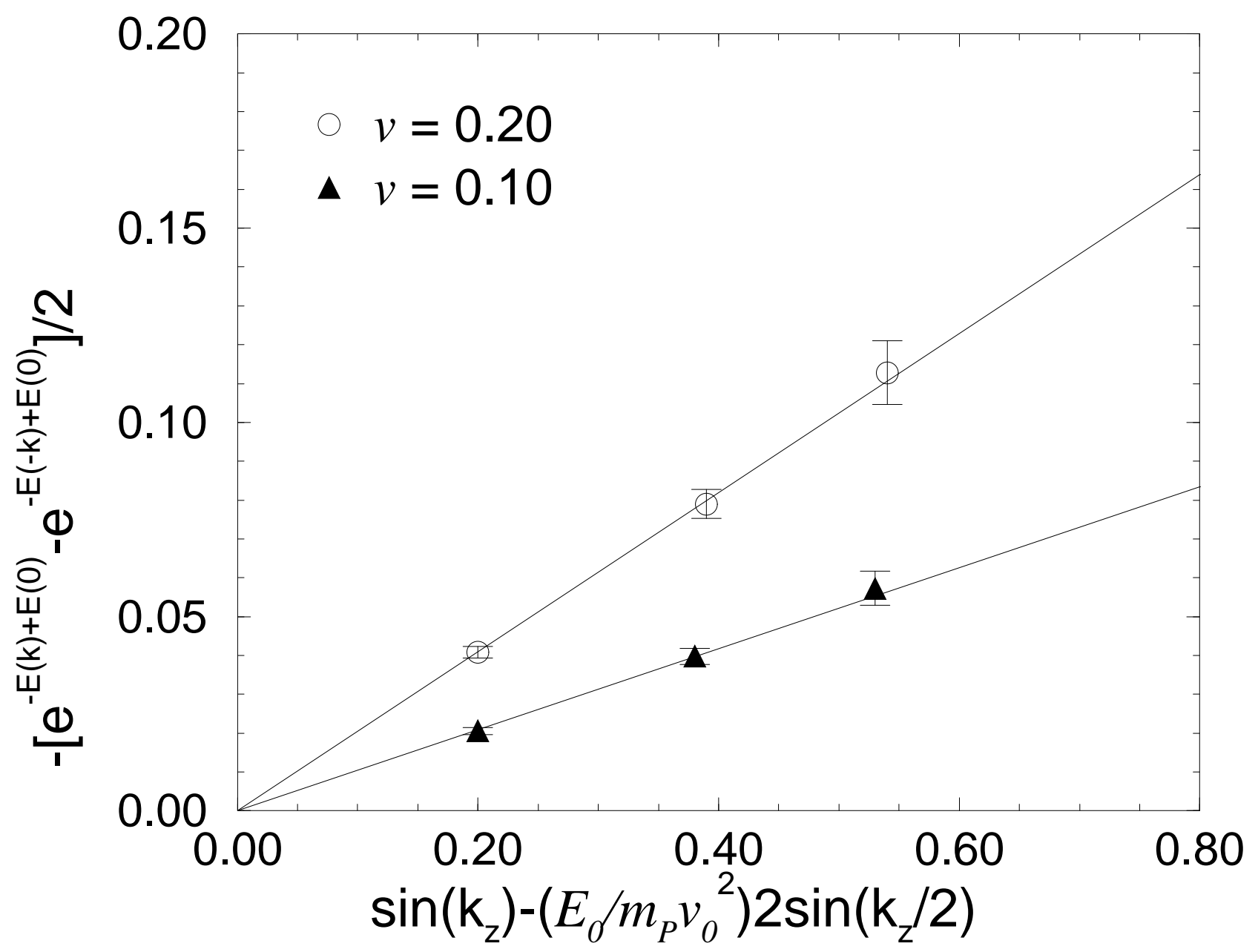




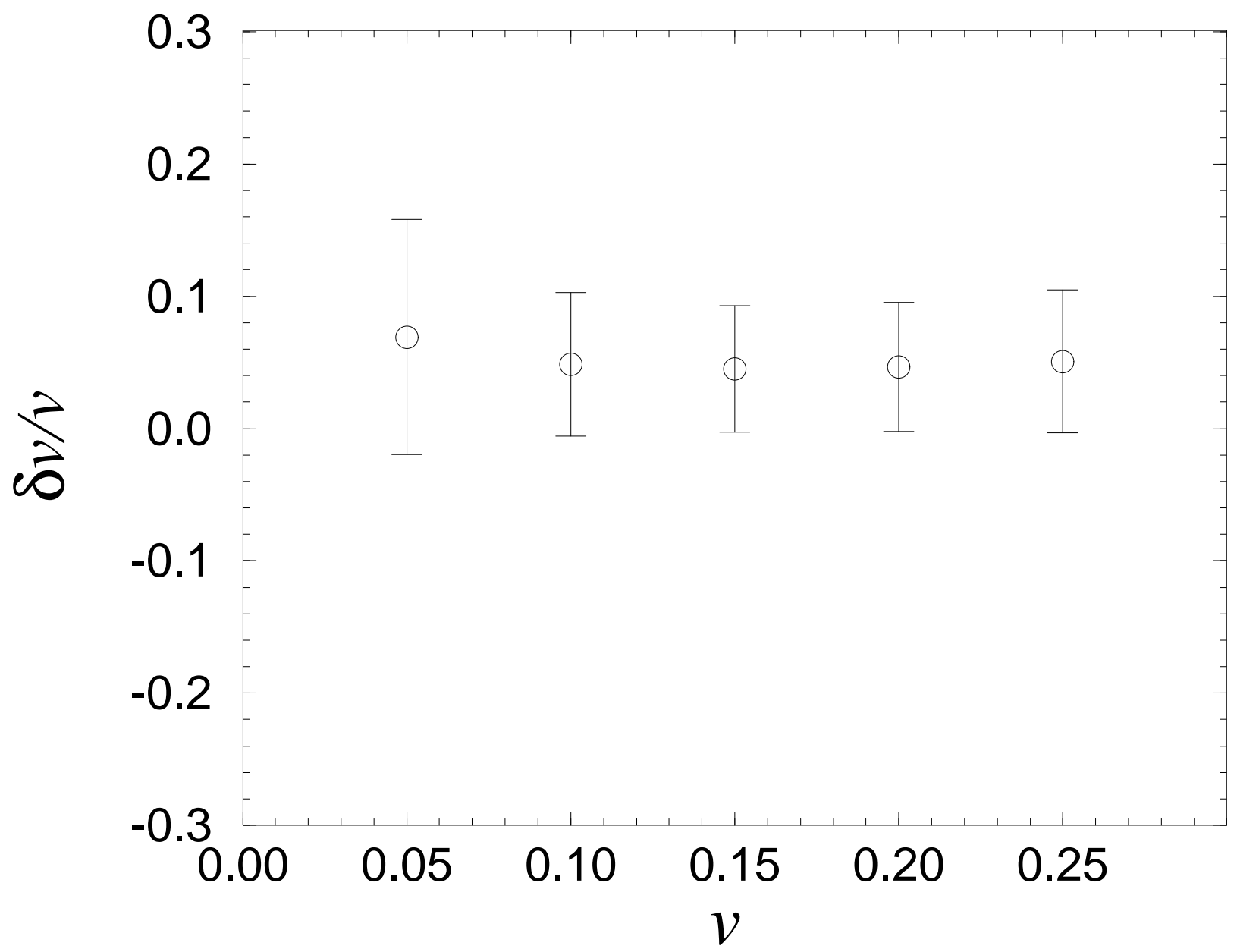




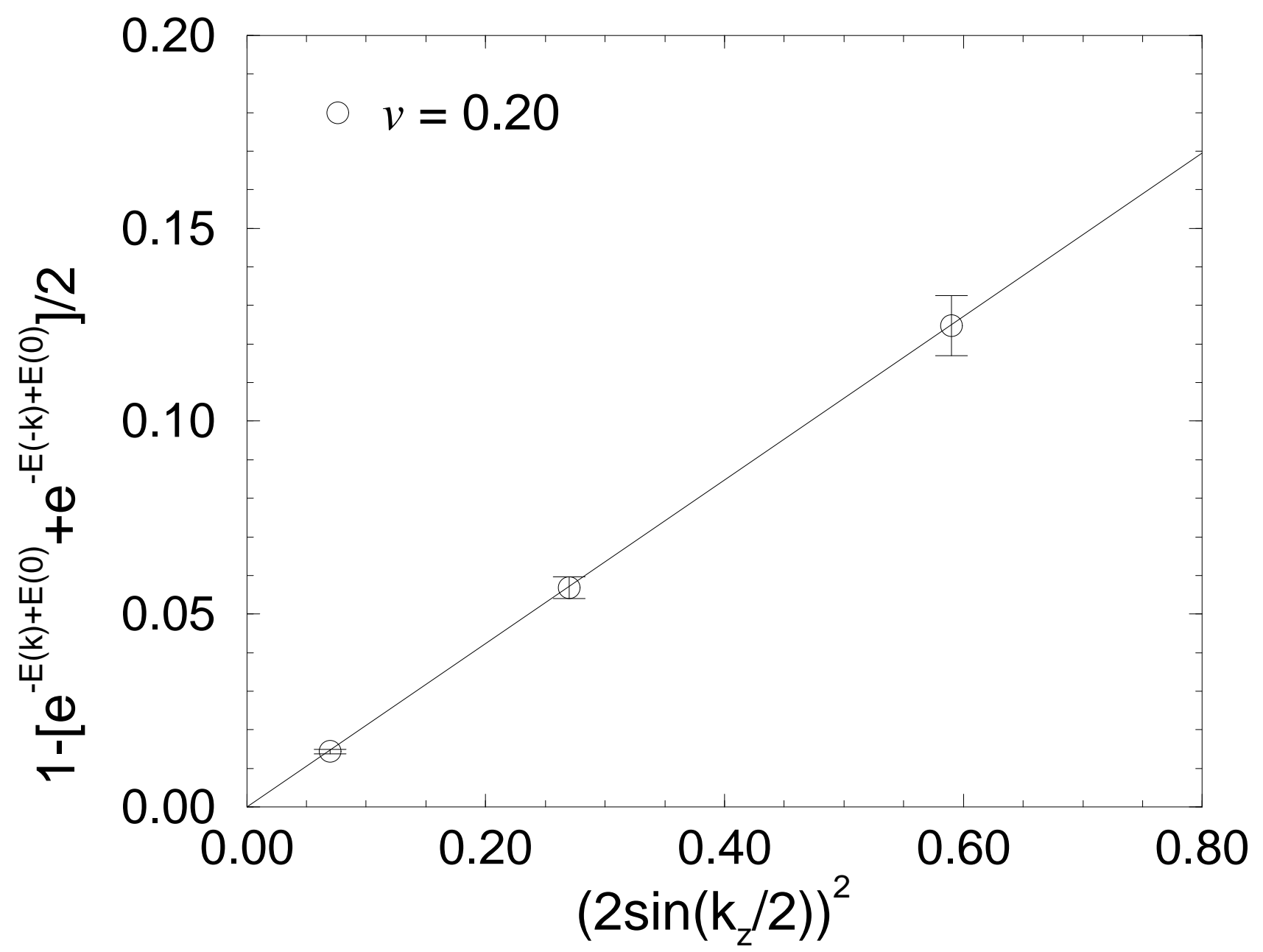




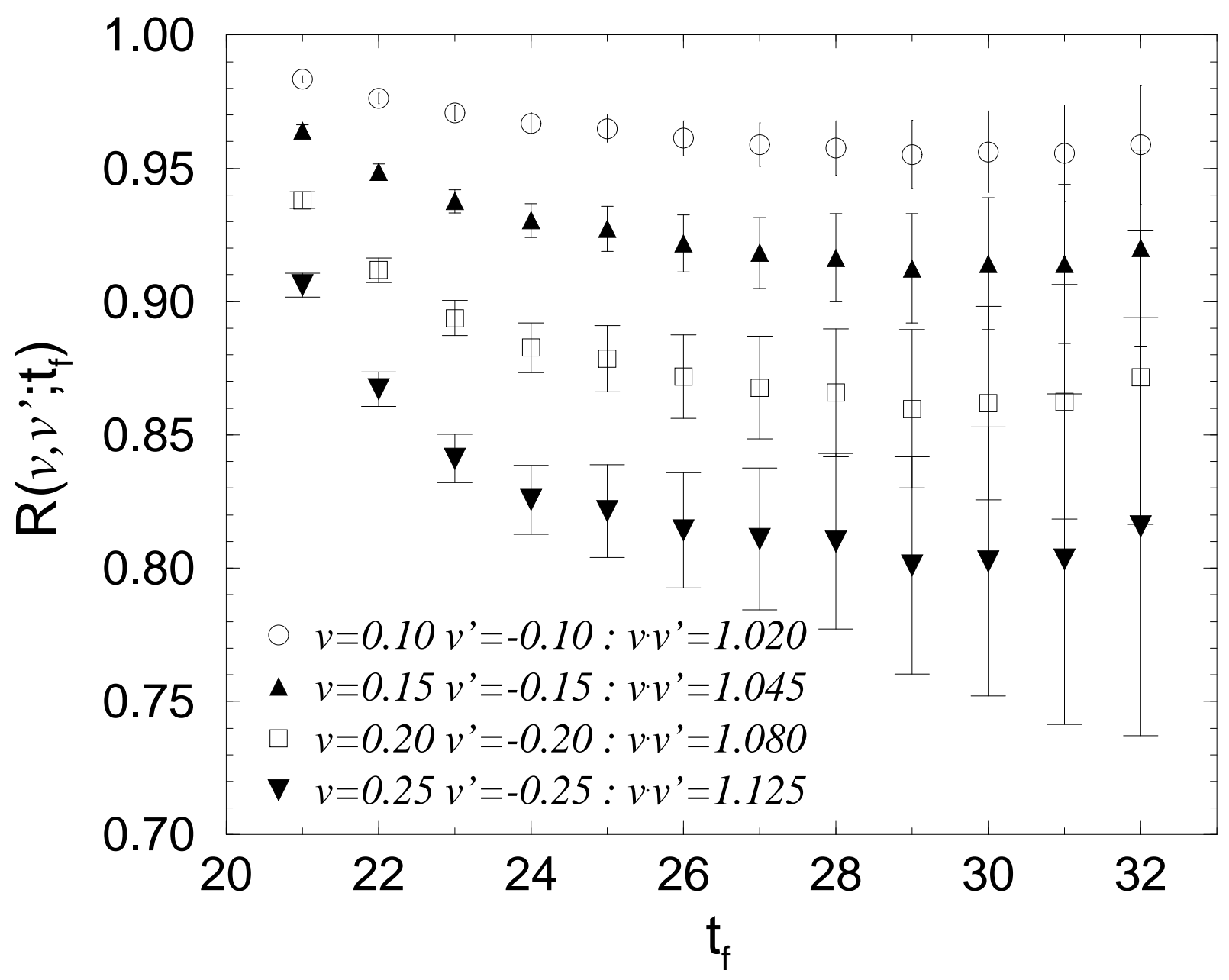




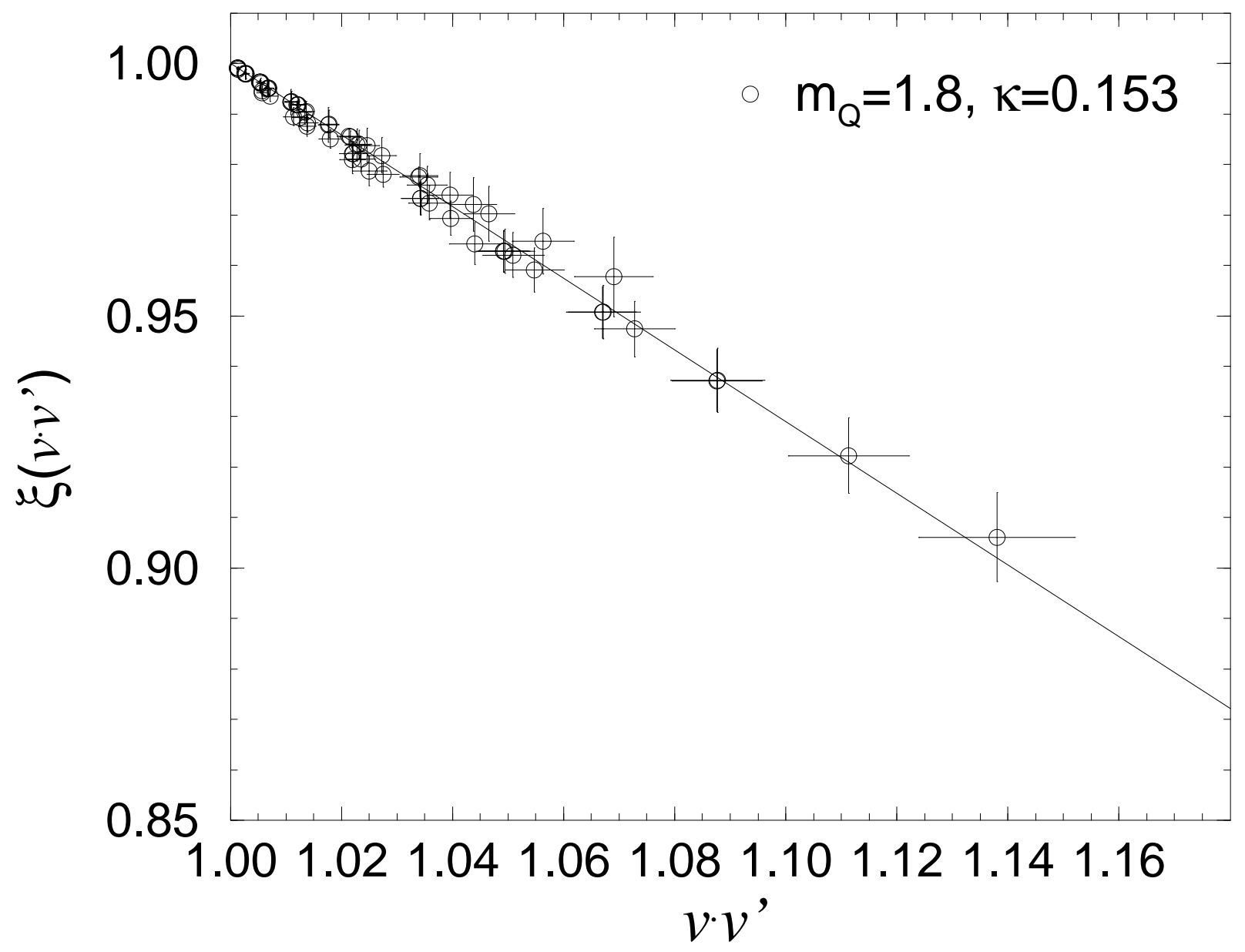




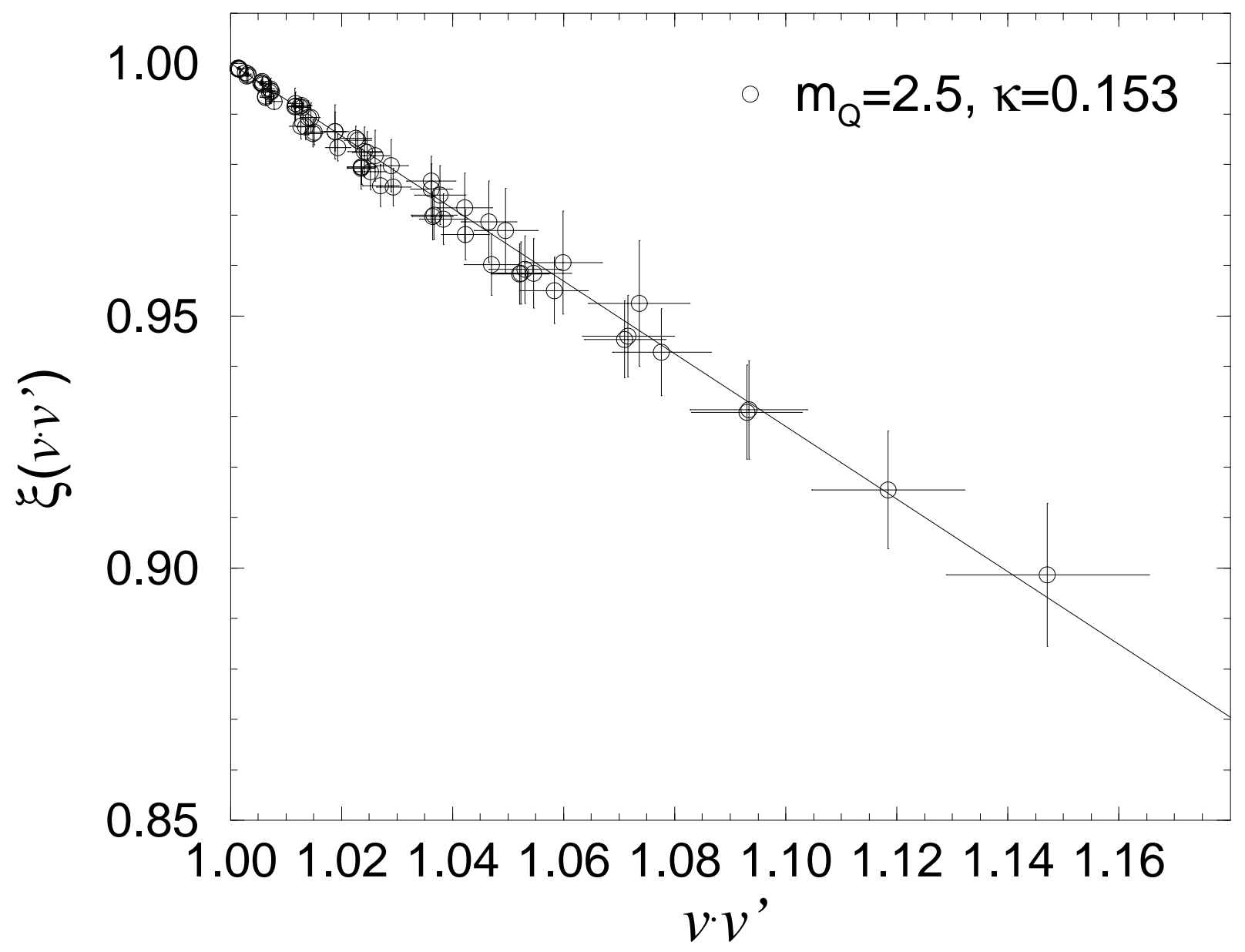




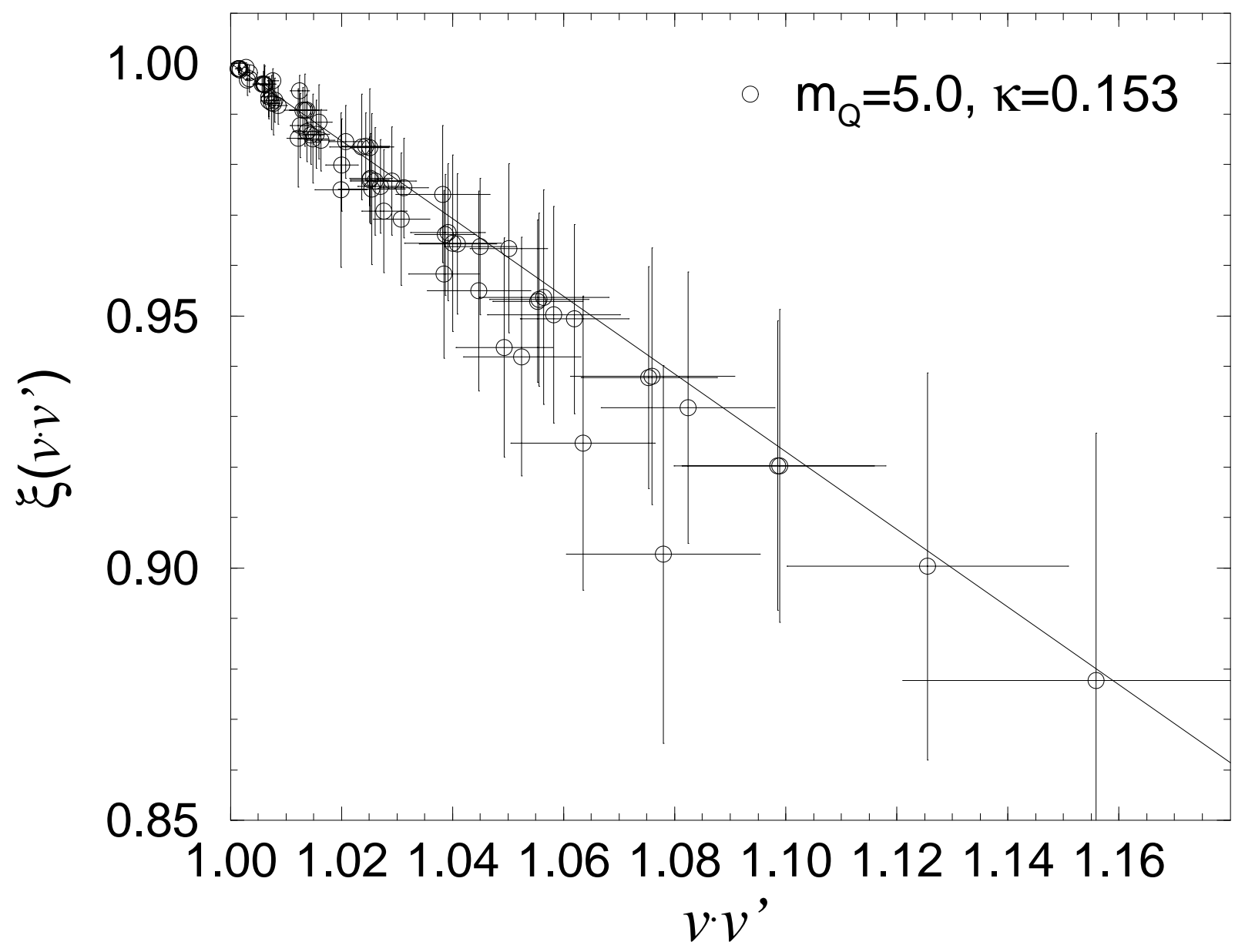




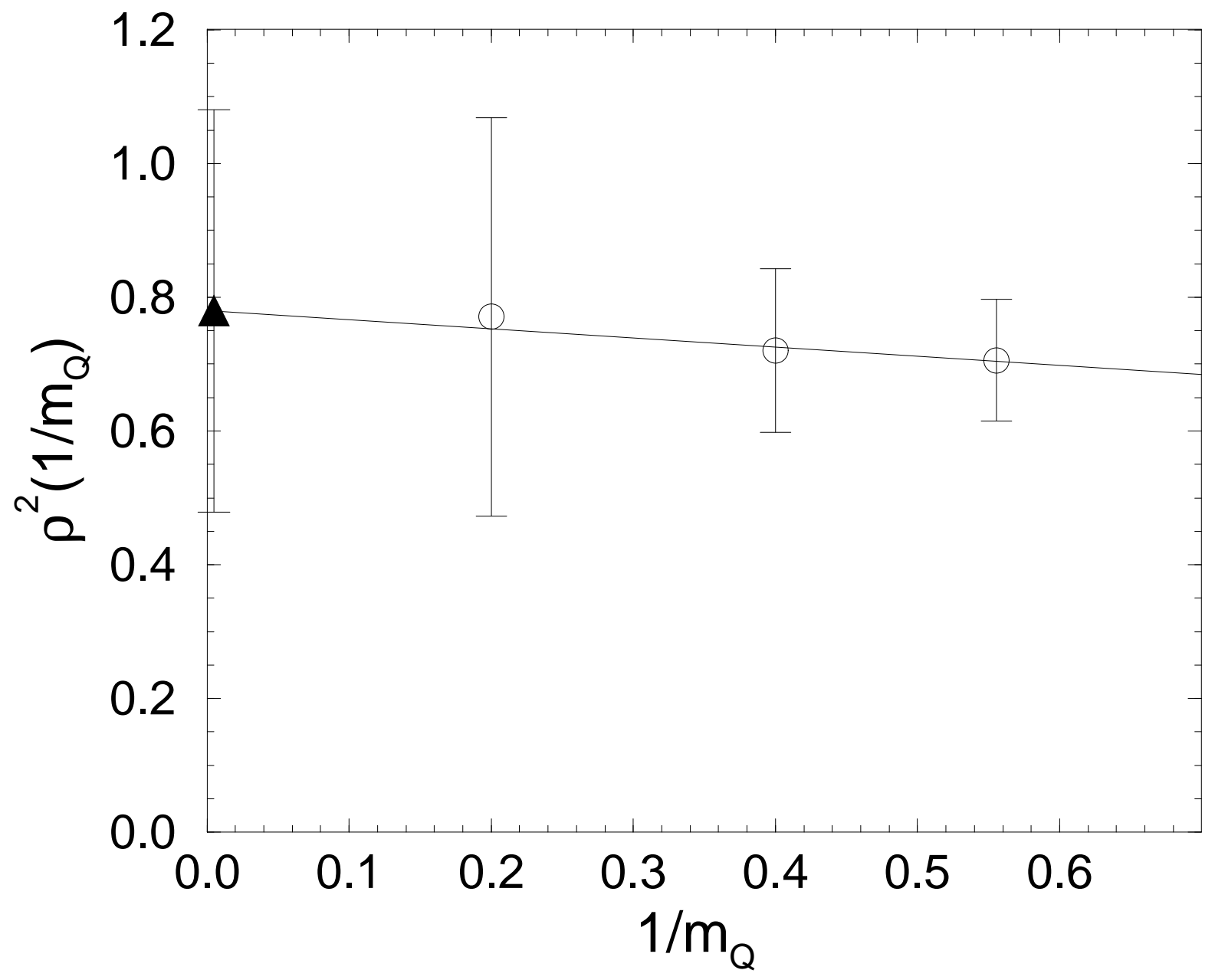

